Ann. Biol. anim. Bioch. Biophys., I975, 15 (4), 739-744.

\title{
INTÉRET DE LA PSEUDOISOCYANINE POUR L'ÉTUDE DES CHROMOSOMES HUMAINS
}

\author{
Laboratoive d'Histologie et Embryologie II, \\ Faculté de Médecine, \\ 27, boulevard Jean-Moulin, \\ 13385 Marseille Cedex 4
}

Anne-Marie VAGNER-CAPODANO, Marie-Hélène DELGROSSI et A. STAHL

\section{RÉSUMÉ}

Le chlorydrate de $\mathrm{N}-\mathrm{N}^{\prime}$ diéthyl-pseudoisocyanine permet l'étude des chromosomes humains mitotiques et méiotiques aussi bien en lumière monochromatique qu'en fluorescence. Couplé avec certains prétraitements et des techniques telles que la dénaturation thermique ménagée, la dénaturation par la formamide, l'incorporation de 5-BUDR, il met en évidence des bandes extrêmement nettes sur les chromosomes. Les tests cytochimiques pratiqués indiquent que la réaction à la pseudoisocyanine présente une certaine spécificité pour l'ADN mais sans exclure totalement le rôle de certaines protéines.

De nombreux fluorochromes ont été préconisés pour la mise en évidence de bandes sur les chromosomes humains. Ils présentent tous le double inconvénient d'un pâlissement rapide sous irradiation ultra-violette, et de la nécessité d'une exploration immédiate des préparations, leur conservation étant pratiquement impossible.

Il n'en est pas de même pour le chlorydrate de diéthyl-pseudoisocyanine dont l'affinité pour la chromatine a été décrite par STERBA (I963). Il présente la formule suivante :<smiles>CCN1C(=Cc2ccc3ccccc3[n+]2CC)C=Cc2ccccc21</smiles>

La pseudoisocyanine est de teinte rouge à l'état de monomère et prend une couleur métachromatique violacée lorsqu'elle se polymérise au contact de fonctions acides. 
SCHÄFFNER (I968) a montré que dans certaines conditions d'utilisation elle colore assez spécifiquement 1'AIN en se polymérisant au contact des groupements phosphate. C'est en partant de cette donnée que nous avons été amenés à utiliser la pseudoisocyanine pour l'étude des chromosomes humains, après incorporation de 5-BUDR (5-Bromodéoxyuridine), après traitement des chromosomes par dénaturation thermique ménagée ou par dénaturation par la formamide (STAHL et VAGNERCapodano, I974; Vagner-Capodano et Stahi, I.974).

\section{MATÉRIEL E'T MÉTHODES} (1964)

Des cultures de lymphocytes humains ont été faites selon la microtechnique de De Grovchy

- Dans certaines d'entre elles, du 5-BUDR a été introduit de façon à obtenir une concentration finale de $200 \mu \mathrm{g} / \mathrm{ml}$ de milieu, 7 heures à I 2 heures avant l'arrêt de la culture (Dutrillaux, 1973).

- Le traitement par dénaturation thermique a été réalisé selon la technique de Dutrillaux et LeJEUne (I97)

- Des préparations ont été traitées durant I heure par la formamide utilisée à la concentration de $50 \mathrm{p}$. 100 dans du $2 \times \mathrm{SSC}$ (sodium saline citrate) à $65^{\circ} \mathrm{C}$.

Ces divers prétraitements ont tous été suivis d'une coloration par la pseudoisocyanine dans les conditions préconisées par Sterba (1963) et Sterba et Schäffner (I965).

Il est procédé d'abord à une méthylation des préparations pendant 5 à 7 heures à $40^{\circ} \mathrm{C}$ dans une solution formée de 1 oo $\mathrm{ml}$ de méthanol absolu et de $3 \mathrm{ml}$ de $\mathrm{SO}_{4} \mathrm{H}_{2}$. Elle est suivie d'une oxydation durant trois minutes par un mélange fraîchement préparé, formé de 8 parties d'eau distillée, une partie de $\mathrm{SO}_{4} \mathrm{H}_{2}$ à $5 \mathrm{p}$. Ioo et une partie de $\mathrm{KMnO}_{4}$ à 2,5 p. Ioo. Les lames sont ensuite rincées à l'eau distillée, puis blanchies pendant ${ }_{5} 5$ secondes dans l'acide oxalique à $3 \mathrm{p}$. roo et enfin rincées à l'eau distillée puis à l'eau courante durant dix minutes. La coloration à la pseudoisocyanine est réalisée durant environ $\mathrm{I}_{4}$ heures dans une solution à $25 \mathrm{mg}$ pour $100 \mathrm{ml}$ d'eau distillée à l'obscurité et à une température de $4^{\circ} \mathrm{C}$. Les lames peuvent être ensuite montées dans le colorant lui-même et lutées au vernis. Nous avons essayé différents procédés pour rendre ces préparations permanentes. Nous avons constaté que l'huile de Rhodorsil $\left({ }^{(}\right)$permet une conservation des lames sans que la coloration ni la fluorescence ne soient altérées.

L'observation des préparations peut se faire

- soit en lumière ordinaire avec interposition d'un filtre monochromatique de $576 \mathrm{~nm}$ de longueur d'onde. Le contraste de phase est inutile;

- soit en lumière ultra-violette : les chromosomes présentent alors une fluorescence jaune très brillante.

\section{OBSERVATIONS}

Sur les préparations obtenues après incorporation de 5 -BUDR et coloration à la pseudoisocyanine, les chromosomes présentent, en lumière monochromatique comme en fluorescence, des bandes rappelant par leur localisation le système des bandes $\mathrm{R}$; mais en fluorescence il semble qu'il y ait une meilleure définition qu'avec l'orange d'acridine, et de plus, des sous-bandes sont apparentes (P1. I, fig. I $a-b$ ).

Après dénaturation thermique et coloration à la pseudoisocyanine les bandes $\mathrm{R}$ apparaissent avec une grande netteté en lumière monochromatique et avec une grande brillance en lumière ultra-violette (P1. I, fig. I c-d).

(1) Huile 64I. V 200 Rhône-Poulenc. 
Après prétraitement par la formamide la coloration par la pseudoisocyanine semble donner une meilleure définition des bandes $\mathrm{R}$ en lumière monochromatique qu'en fluorescence (P1. I, fig. I $e-f$ ).

Nous avons entrepris une étude cytochimique de la réaction de la pseudoisocyanine pour tenter d'identifier les substances colorées par ce fluorochrome. Ces tests ont été pratiqués sur des préparations n'ayant subi aucun prétraitement (tab1. I).

TABLEAU I

\begin{tabular}{|c|c|c|c|c|}
\hline Tests cy tochimiques & $\begin{array}{l}\text { Température } \\
\left({ }^{\circ} \mathrm{C}\right)\end{array}$ & Temps & Substrats & $\begin{array}{l}\text { Coloration } \\
\text { par la PIC }\end{array}$ \\
\hline $\begin{array}{c}\text { RNase } \\
0,01 \text { p. } 100 \text { dans } \mathrm{H}_{2} \mathrm{O} \text { dist. }\end{array}$ & 37 & $\begin{array}{l}1 \mathrm{~h} \\
2 \mathrm{hh} \\
3 \mathrm{~h} \\
4 \mathrm{~h}\end{array}$ & ARN & + \\
\hline $\begin{array}{c}\text { DNase } \\
0,02 \text { p. } 100 \text { dans } \mathrm{MgSO}_{4} \\
\text { à } \mathrm{pH}=6,3\end{array}$ & 37 & $\begin{array}{ll}1 \mathrm{~h} \\
2 \mathrm{~h} \\
3 \mathrm{~h} \\
4 \mathrm{~h}\end{array}$ & $\mathrm{ADN}$ & - \\
\hline $\begin{array}{c}T C A \\
5 \text { p. } 100 \text { dans } \mathrm{H}_{2} \mathrm{O} \text { dist. }\end{array}$ & 90 & $\begin{array}{r}5 \mathrm{mn} \\
10 \mathrm{mn} \\
15 \mathrm{mn} \\
20 \mathrm{mn}\end{array}$ & $\begin{array}{l}A R N+A D N \\
A R N+A D N \\
A R N+A D N \\
A R N+A D N\end{array}$ & $\begin{array}{l} \pm \\
\pm \\
-\end{array}$ \\
\hline $\mathrm{HCl}\left\{\begin{array}{l}1 \mathrm{~N} \mathrm{HCl} \\
2 \mathrm{~N} \mathrm{HCl}\end{array}\right.$ & $\begin{array}{r}60 \\
60 \\
60 \\
4\end{array}$ & $\begin{array}{l}8 \mathrm{mn} \\
15 \mathrm{mn} \\
30 \mathrm{mn} \\
16 \mathrm{~h}\end{array}$ & $\begin{array}{c}\text { ARN } \\
\text { ARN } \\
\text { ARN }+ \text { ADN } \\
\text { ARN }\end{array}$ & $\begin{array}{l}+ \\
+ \\
- \\
+\end{array}$ \\
\hline $\begin{array}{c}\text { Pepsine } \\
0,04 \text { p. } 100 \text { dans tampon } \\
\text { Acćtate } / \mathrm{HCl} \text { à } \mathrm{pH}=2\end{array}$ & 37 & $\begin{array}{l}15 \mathrm{mn} \\
20 \mathrm{mn} \\
40 \mathrm{mn}\end{array}$ & Protéines & $\begin{array}{l}+ \\
+ \\
+\end{array}$ \\
\hline $\begin{array}{c}\text { Trypsine } \\
0,01 \text { p. } 100 \text { dans } \mathrm{H}_{2} \mathrm{O} \text { dist. }\end{array}$ & 37 & $\begin{array}{l}15 \mathrm{~s} \\
30 \mathrm{~s} \\
1 \mathrm{mn} \\
3 \mathrm{mn} \\
5 \mathrm{mn} \\
10 \mathrm{mn} \\
30 \mathrm{mn}\end{array}$ & Protéines & $\begin{array}{l}+ \\
+ \\
+ \\
+ \\
+ \\
+ \\
+\end{array}$ \\
\hline $\begin{array}{c}\text { Pronase } \\
0,005 \text { p. } 100 \text { dans } \mathrm{H}_{2} \mathrm{O} \text { dist. }\end{array}$ & 37 & $\begin{array}{r}1 \mathrm{mn} \\
3 \mathrm{mn} \\
5 \mathrm{mn} \\
10 \mathrm{mn} \\
30 \mathrm{mn}\end{array}$ & Protéines & $\begin{array}{l}+ \\
+ \\
+ \\
+ \\
+\end{array}$ \\
\hline
\end{tabular}

Après ribonucléase, la coloration à la pseudoisocyanine persiste (P1. II, fig. $2 a$ ) alors qu'elle est négative par l'action de la désoxyribonucléase (P1. II, fig. $2 b$ ) ; dans ce cas les chromosomes ne sont plus colorés ; leur "fantôme " peut être retrouvé en contraste de phase. 
L'acide trichloracétique utilisé à des temps très courts réduit l'intensité de la coloration par la pseudoisocyanine, et la fait disparaître comme dans le cas de la désoxyribonucléase, lorsqu'il agit plus longtemps.

A certaines températures et pour des temps déterminés l'hydrolyse par l'acide chlorhydrique laisse la coloration intacte tant que l'ADN n'est pas altéré.

La coloration par la pseudoisocyanine persiste après traitement par différentes enzymes protéolytiques : pepsine, trypsine, pronase. Cette constatation n'est pas favorable à un rôle des protéines, en particulier des histones, dans la coloration des chromosomes par la pseudoisocyanine. Toutefois pour STERBA et SCHÄFFnER il ne faut pas exclure totalement le rôle des protéines en particulier celles portant des groupements - SH et - SS dans la réaction de coloration par la pseudoisocyanine.

Le résultat de ces tests permet d'attribuer à la pseudoisocyanine une certaine spécificité pour l'ADN. Cette spécificité n'atteint pas celle de la réaction de Feulgen mais la coloration par la pseudoisocyanine est plus sensible que cette dernière et permet la détection de faibles quantités d'ADN. Son utilisation dans les ovocytes humains fournit des images d'une grande finesse lorsque les chromosomes commencent à se déspiraliser au stade diplotène de la méiose (P1. III, fig. $3 a-b$ ).

La pseudoisocyanine se prête à un grand nombre de techniques de bandes. Dans tous les cas les préparations peuvent être examinées en lumière ultra-violette. Elles présentent une fluorescence intense qui résiste très longtemps à l'irradiation contrairement aux autres fluorochromes. Ceci permet une étude à la fois prolongée et renouvelée des préparations et offre l'avantage de pouvoir utiliser une émulsion à grain très fin pour la microphotographie.

Cette fluorescence, en outre, persiste durant plusieurs mois lorsqu'on conserve les préparations au froid, à l'abri de la lumière. Les lames peuvent être colorées si cela est nécessaire au bout d'un certain temps, elles retrouvent alors leur aspect initial. La coloration métachromatique n'étant pas altérée après irradiation, il est possible de réexaminer les lames en lumière ordinaire sans contraste de phase, simplement avec un filtre ou un monochromateur à une longueur d'onde de $576 \mathrm{~nm}$.

Ces propriétés confèrent à la pseudoisocyanine une place de choix parmi les techniques utilisées pour l'étude des chromosomes.

Colloque D. G. R. S. T., Biologie de la Procréation.

Paris, $7-8$ mars 1975 .

\section{REMERCIEMENTS}

La longueur d'onde $57^{6} \mathrm{~nm}$ correspond au pic d'absorption mesuré sur nos préparations par M. le Docteur VILTER que nous remercions pour son aide.

Ce travail a bénéficié de l'aide de la D. G. R. S. T., contrat no $7^{2-7^{-003} \text { I }}$

\section{SUMMARY}

\section{THE STAINING OF HUMAN CHROMOSOMES WITH PSEUDOISOCYANINCHLORIDE}

The chlorydrate of $\mathrm{N}-\mathrm{N}^{\prime}$ diethyl-pseudoisocyanine allows the study of mitotic and meiotic human chromosomes as well in monochromatic light as in fluorescence. Joined with some pretreatments and methods such as heat denaturation, formamide denaturation, and 5-BUDR 
incorporation, the pseudoisocyanine shows typical banding on the chromosomes. Cytochemical tests indicate that the pseudoisocyanine reaction shows some specificity for DNA but the role of some proteins cannot be excluded.

\section{RÉFÉRENCES BIBLIOGRAPHIQUES}

Dutrillaux B., Lejeune J., I97I. Sur une nouvelle technique d'analyse du caryotype humain. C. $R$. Acad. Sci. (Paris), D, 272, 2638-2640.

Dutrillaux B., Laurent C., Couturier J., Lejeune J., 1973. Coloration des chromosomes humains par l'acridine orange après traitement par le 5-bromodéoxyuridine, C. R. Acad. Sci. (Paris), D, 276, 3I79-3I8r.

Grouchy J. (de), Roubin M., Passage E., I964. Microtechnique pour l'étude des chromosomes humains à partir d'une culture de leucocytes sanguins. Ann. Génét., ry, 45-47.

SchäfFner H., I968. Untersuchugen an Oozytenkernen von Amphibien und Teleosteern mit Pseudoisozyanin. Histochemie, 13, 346-378.

Stahl A., Vagner-Capodano A. M., I974. Mise en évidence des bandes des chromosomes humains en lumière ordinaire et en fluorescence après coloration par la pseudoisocyanine. C. R. Acad. Sci. (Paris), 278, 2987-2989.

Sterba G., I963. Ankündigung eines neuen histochemischen DNS-Nachweises mit hoher, die Feulgenreaktion übertreffender Empfindlichkeit. Acta Biol. Med. Germ., 10, 694-7oo.

Sterba G., SchäFFnER H., I965. Fluoreszenzmikroskopischer Nachweis der DNS in Lampenbürstenchromosomen mit NN'-Diäthylpseudoisozyaninchlorid. Histochemie, 5, 260-278.

Vagner-Capodano A. M., Staht, A., 1974. Human chromosome banding revealed by N-N' diethylpseudoisocyaninchloride after heat denaturation in light and fluorescence microscopy. Leiden Chromosome Conference, July i5-17. 


\section{PLANCHE I}

Chromosomes humains colorés par la pseudoisocyanine et observés successivement en lumière monochromatique et en lumière ultraviolette.

$a$ et $b$ : après traitement par le 5-BUDR

$c$ et $d$ : après dénaturation thermique ménagée

$e$ et $f$ : après traitement par la formamide 

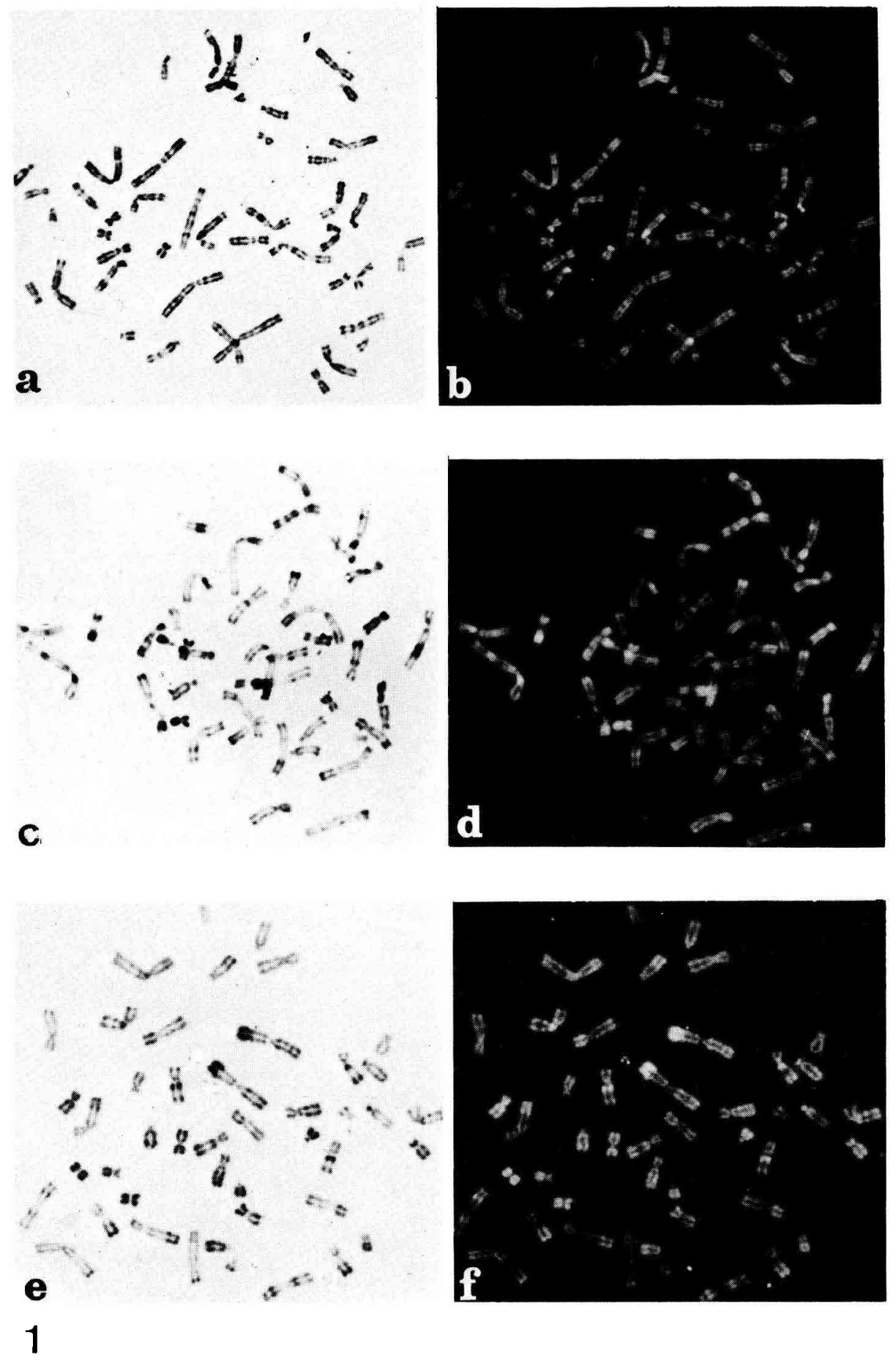


\section{PLANCHE II}

a : Métaphase observée après traitement par la ribonucléase et coloration par la pseudoisocyanine. L'intensité de la coloration n'est pas altérée.

b : Mitose photographiée en contraste de phase après traitement par la désoxyribonucléase et coloration par la pseudoisocyanine. La réaction est négative. 


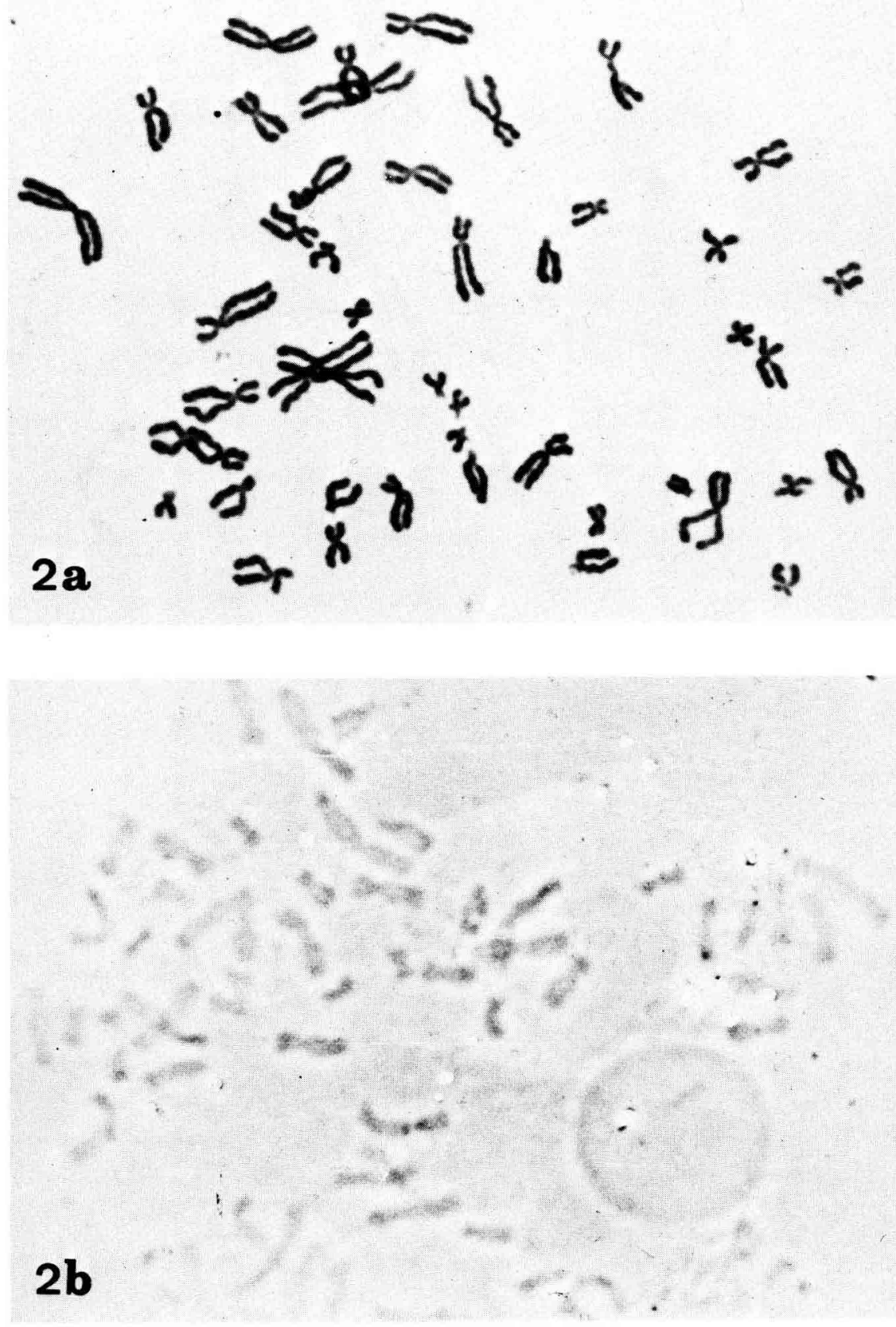




\section{PLANCHE III}

a : Ovocyte humain : stade diplotène coloré par la pseudoisocyanine :

a : observé en lumière monochromatique

b : même noyau observé en lumière ultra-violette. 

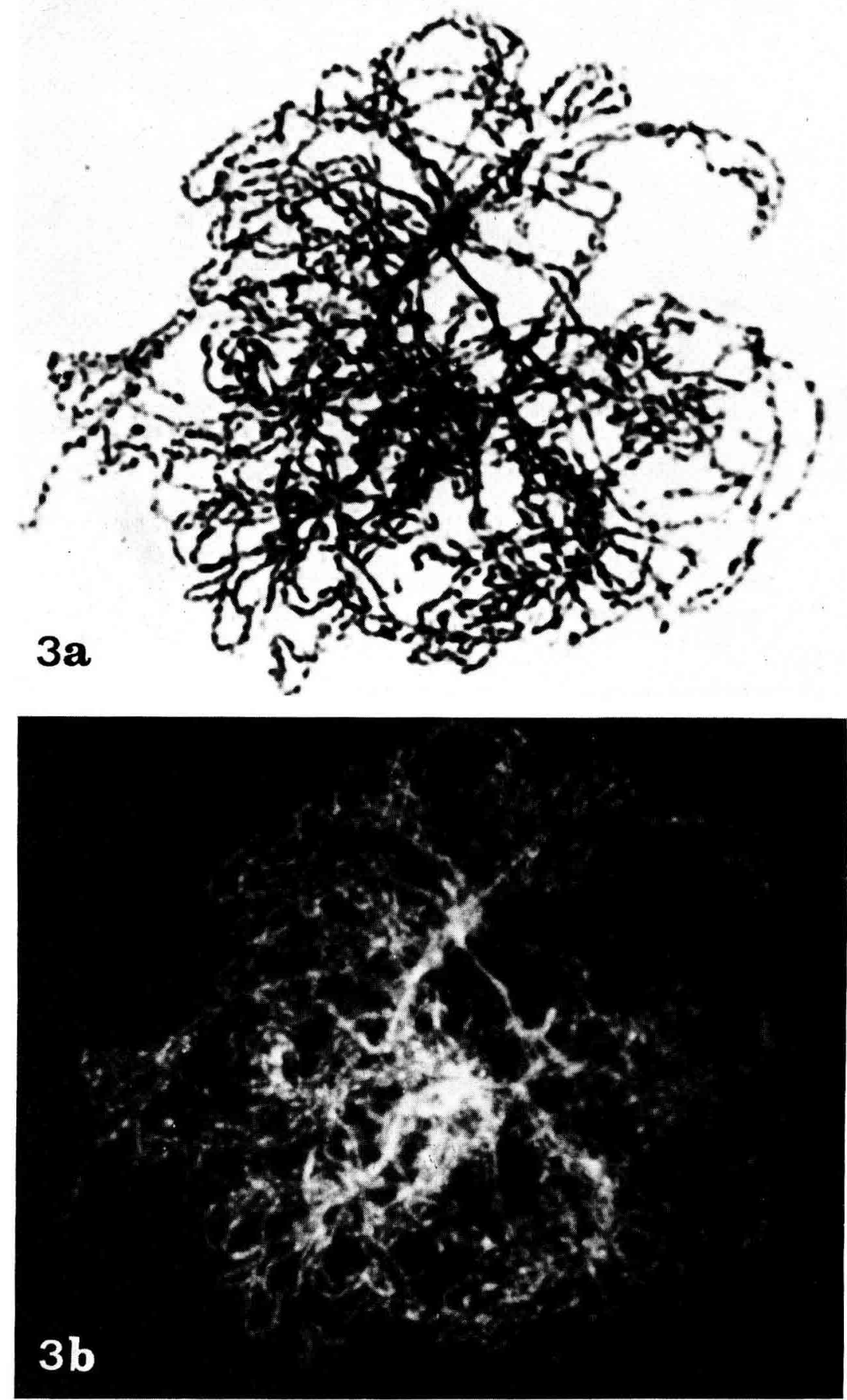